\title{
The Inflammatory Transcription Factor C/EBP $\beta$ Plays a Critical Role in Cardiac Fibroblast Differentiation and a Rat Model of Cardiac Fibrosis Induced by Autoimmune Myocarditis
}

\author{
Xiu Li, ${ }^{1, *}$ MD, Menghua Sun, ${ }^{1, *}$ MD, Suzhen Men, ${ }^{1}$ MD, Yanan Shi, ${ }^{1}$ MD, Lijuan Ma, ${ }^{1}$ MD, \\ Yongqiang An, ${ }^{1} \mathrm{MD}$, Yaqing Gao, ${ }^{1} \mathrm{MD}$, Hui Jin, ${ }^{2} \mathrm{MD}$, Wei Liu, ${ }^{1} \mathrm{MD}$ and Zuoyi Du, ${ }^{2} \mathrm{MD}$
}

\begin{abstract}
Summary
The aim of the present study was to investigate the mechanisms of CCAAT/enhancer-binding protein $\beta$ (C/ $\mathrm{EBP} \beta)$ in cardiac myofibroblast (CMF) differentiation and in a rat model of cardiac fibrosis induced by experimental autoimmune myocarditis (EAM).

In vitro studies performed in primary neonatal rat CMF revealed that silencing of C/EBP $\beta$ expression (via lentiviral mediated shRNA strategies) was sufficient to reduce C/EBP $\beta$ mRNA and protein levels as well as to decrease the expressions of actin cytoskeletal proteins, cofilin, and filamin A (FLNA). TGF $\beta$ increased IL-1 $\beta$, IL-6 and TNF-a production in cardiac fibroblasts $(\mathrm{CF})$, while C/EBP $\beta$ knockdown reduced the secretion of these inflammatory mediators. In vivo studies performed in rats exhibiting EAM revealed that lentiviralmediated silencing of $\mathrm{C} / \mathrm{EBP} \beta$ was sufficient to reduce the expression of $\mathrm{C} / \mathrm{EBP} \beta$ as well as inflammation and fibrosis in the hearts of EAM rats, when compared to controls. Echocardiography further revealed that $\mathrm{C} / \mathrm{EBP} \beta$ knockdown was sufficient to significantly improve cardiac dimensions and function in EAM rats. Immunohistochemical results showed that C/EBP $\beta$ knockdown attenuated the expression of C/EBP $\beta$ protein as well as the expressions of collagen I, collagen III, MMP-2, MMP-9, and $\alpha$-SMA in heart tissue sections from rats in the $\mathrm{EAM}+$ Lenti-shC/EBP $\beta$ group.

Strategies targeted at inhibiting $\mathrm{C} / \mathrm{EBP} \beta$ expression can be potentially exploited to regulate cofilin and FLNA expression, thereby regulating actin polymerization/depolymerization, cytoskeleton rearrangement, and $\mathrm{CF}$ differentiation into $\mathrm{CMF}$ and the production of inflammatory cytokines. C/EBP $\beta$ knock down reduces the degree of inflammation-mediated myocardial fibrosis in a rat model of EAM.
\end{abstract}

(Int Heart J 2018; 59: 1389-1397)

Key words: Cardiac remodeling, Inflammation, Extracellular matrix, Myofibroblast, $\alpha-S M A$

$\mathrm{M}$ yocardial fibrosis is a key cause of heart failure, malignant arrhythmias and other cardiovascular events. ${ }^{1-3)}$ It is characterized as an accumulation of collagen fibers; a significant increase in collagen concentration, and a change in the composition of collagen components in myocardial tissue. Myocardial fibrosis is a nearly inevitable outcome in end-stage cardiovascular disease, which results in cardiac structural remodeling due to excess extracellular matrix (ECM) ${ }^{4 \cdot 6)}$ The ECM is mainly composed of type I and type III collagen. Approximately $85 \%$ of total myocardial collagen is type I, while type III collagen accounts for $11 \%$ of the total collagen protein. ${ }^{7.8)}$

During fibrosis, quiescent cardiac fibroblasts (CF) become activated and differentiate into cardiac myofibro- blasts (CMF), which typically express $\alpha$-smooth muscle actin $(\alpha$-SMA) and acquire the ability to proliferate, migrate, and produce ECM. ${ }^{1,9,10)}$ Fibroblast to myofibroblast differentiation is a key process during cardiac remodeling. Transforming growth factor-beta 1 (TGF- $\beta 1$ ) plays an important role in the transdifferentiation of $\mathrm{CF}$ into CMF, which is an important mediator of myocardial fibrosis. ${ }^{11}$ Independent studies have also suggested myocardial inflammation is a mechanism that also leads to the development of cardiac fibrosis, ${ }^{12-14)}$ and is intimately linked to myocardial fibrosis leading to heart failure. ${ }^{6,13)}$

Compelling evidence suggests that $\mathrm{C} / \mathrm{EBP} \beta$ is an important regulator of cell proliferation, differentiation and apoptosis, ${ }^{15,16)}$ and can also significantly attenuate fibrosis in the liver and lung; ${ }^{17,18)}$ however, the impact and mecha-

From the ${ }^{1}$ Department of Cardiology, the Fourth Affiliated Hospital, Harbin Medical University, Harbin, PR. China and ${ }^{2}$ Department of Cardiology, The Second People's Hospital of Guangdong Province, Guangdong, PR. China.

*These authors contributed equally to this work.

This study was supported by NSFC (81270310), Harbin Medical University Innovation Fund for postgraduate students (YJSCX2015-51HYD) Innovation Fund from Harbin Medical University and Harbin science and technology innovation talents (2016RAYBJ005).

Address for correspondence: Wei Liu, MD, Department of Cardiology, The Fourth Affiliated Hospital, Harbin Medical University, No. 37 YiYuan Street, Harbin 150001, PR. China. E-mail: doctor_liuwei@126.com or Zuoyi Du, MD, Department of Cardiology, The Second People's Hospital of Guangdong Province, No. 466 Xingang East Road, Haizhu District, Guangzhou, Guangdong 510317, PR China. E-mail: duzuoyi888@126.com

Received for publication November 15, 2017. Revised and accepted March 13, 2018.

Released in advance online on J-STAGE October 10, 2018.

doi: 10.1536/ihj.17-446

All rights reserved by the International Heart Journal Association. 


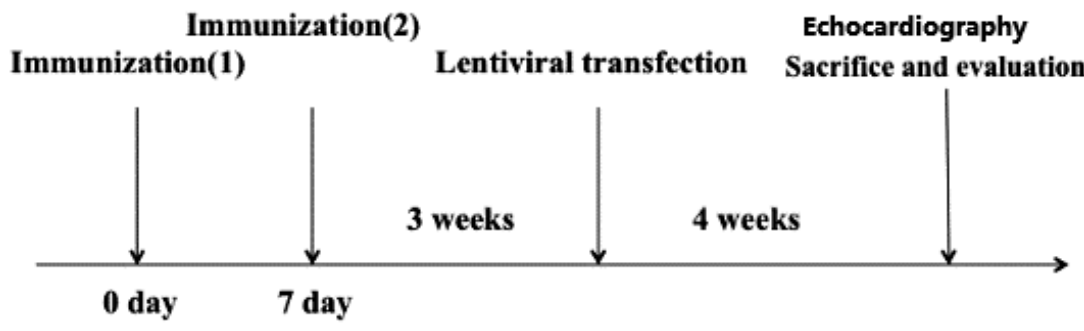

Figure 1. Protocol. The immunizations were performed on 0 day and 7 day. Lentiviral transfection was performed 3 weeks after the second immunization and echocardiography was performed 4 weeks after the lentiviral transfection.

nisms of $\mathrm{C} / \mathrm{EBP} \beta$ in $\mathrm{CMF}$ and $\mathrm{CF}$ remain elusive. The aim of the present study was to examine the effects of $\mathrm{C} /$ $\mathrm{EBP} \beta$ knockdown in primary neonatal rat $\mathrm{CF}$ and a rat model of cardiac fibrosis induced by experimental autoimmune myocarditis (EAM) using a lentiviral shRNA approach.

\section{Methods}

C/EBP $\beta$ lentiviruses: Lentiviruses containing small hairpin RNA (shRNA) (Lenti-shC/EBP $\beta$ ) directed against $\mathrm{C} /$ EBP $\beta$ (shRNA sequence: GAAGAAACGTCTATGTGTA) were obtained from Genechem (Shanghai, China). Lentivirus containing vector was used as control.

C/EBP $\beta$ lentiviral studies in cardiac myofibroblast cultures and rat EAM model: For in vitro studies, rat cardiac fibroblasts were isolated from 3-day-old neonatal Sprague-Dawley rat pups using a differential adhesion method as described previously. ${ }^{19)}$ Cells were centrifuged after digestion and suspended in Dulbecco's Modified Eagle's Medium (DMEM; Hyclone, Logan, CT) supplemented with $10 \%$ fetal bovine serum (FBS, Hyclone, Logan, CT), and then plated on dishes in DMEM at $37^{\circ} \mathrm{C}$ and $5 \% \mathrm{CO}_{2}$. After 1 hour, the adhered $\mathrm{CF}$ were cultured in DMEM containing 10\% fetal calf serum (hyclone). The $\mathrm{CF}$ were subdivided into 4 groups: (1) Control group; $\mathrm{CF}$ with no treatment, (2) Mock group (TGF $\beta 1$ group); $\mathrm{CF}$ treated with TGF- $\beta 1$ (10 ng/mL) for 72 hours (to induce the transition from cardiac fibroblasts to myofibroblasts), (3) Vector group (TGF- $\beta 1+$ lentiviral vector group); $\mathrm{CF}$ treated with TGF- $\beta 1(10 \mathrm{ng} / \mathrm{mL})$ and infected with a control lentivirus for 72 hours, and (4) Lenti-shC/EBP $\beta$ group (TGF- $\beta 1+$ Lenti-shC/EBP $\beta$ group); CF treated with TGF$\beta 1(10 \mathrm{ng} / \mathrm{mL})$ and infected with a lentivirus harboring a shRNA to $\mathrm{C} / \mathrm{EBP} \beta$ for 72 hours, in order to investigate the effect of Lenti-shC/EBP on the transformation of $\mathrm{CF}$ into $\mathrm{CMF}$.

For in vivo studies, the animals were randomly divided into 4 groups ( $n=6$ rats/group) (1): Saline group; injected in the footpad with saline alone, (2) Negative control group (EAM group); injected in the footpad with the emulsion and developed myocarditis, (3) Positive control group (EAM + Lentiviral Vector); injected in the footpad and developed myocarditis and subsequently infected at day 28 with a control lentivirus, and (4) Experimental group (EAM + Lenti-shC/EBP $\beta$ ); rats developed myocarditis and subsequently infected with a lentivirus con- taining a shRNA to C/EBP $\beta$. For lentivirus administration, the rats were anaesthetized with chloral hydrate $(0.3 \mathrm{~mL} /$ $100 \mathrm{~g}$ body weight) and immobilized to isolate the femoral vein for lentiviral injection. Rats were injected in the femoral vein with $0.2 \mathrm{~mL}$ of a control lentivirus $\left(10^{8}\right)$ (SonoVue as carrier) or a lentivirus harboring shRNA to $\mathrm{C} / \mathrm{EBP} \beta\left(10^{8}\right)$ (SonoVue as carrier) using an ultrasoundtargeted microbubble gene therapy technique. ${ }^{20)}$ Rats that did not undergo lentiviral injection were not subjected to transthoracic ultrasound irradiation (Figure 1).

In vivo study:

Animals and immunization protocol These studies were performed at Harbin Medical University and approved by the Experimental Animal Care and Use Committee of Harbin Medical University. Twenty-four inbred male Lewis rats (5 weeks, 180-200 g) were purchased from Beijing Vital River Laboratory Animals Co. Ltd. (Beijing, China) and housed under specified pathogen free conditions.

Cardiac myosin purified from porcine heart $(9.8 \mathrm{mg} /$ L; Sigma, CO) was emulsified with an equal volume of complete Freund's adjuvant (CFA; Sigma, CO), which was supplemented with Mycobacterium tuberculosis H37 RA (Difco, USA). Rats were injected with either saline (Saline) or this emulsion $(0.2 \mathrm{~mL}$; EAM) in the footpad on days 0 and 7 to induce an experimental autoimmune myocarditis model, as described previously. ${ }^{21)}$

Echocardiography Four weeks after lentiviral infection, echocardiography was performed on the rats as described previously. ${ }^{22,23)}$ Briefly, the rats were anesthetized with chloral hydrate and subjected to transthoracic echocardiograms using a General Electric (GE, Vivid-5) imaging system equipped with a 1.7-3.6 MHz multi-frequency transducer in order to measure interventricular septal wall thickness at end diastole (IVS), left ventricular posterior wall thickness (LVPW), left ventricular end diastolic diameter (LVEDD), left ventricular end systolic diameter (LVESD), ejection fraction (EF), and fractional shortening (FS).

Cardiac histology with HE staining and Masson's staining Heart tissue was harvested from anesthetized rats. The left ventricles were separated and sectioned transversely into 4 slices from apex to base. All slices were formalin-fixed, paraffin-embedded, and sectioned into $5 \mu \mathrm{m}$ thick sections. Deparaffinized rat heart tissue sections were then subjected to hematoxylin and eosin staining or Masson's trichrome stain according to the manufacturer's protocols. 
Table I. Primers for Quantitative RT-PCR

\begin{tabular}{lc}
\hline \multicolumn{1}{c}{ Name } & Sequence (5'-3') \\
\hline C/EBP $\beta$ F & GCCAAGAAGGCGGTGGACAA \\
C/EBP $\beta$ R & CAAGTTCCGCAGCGTGCTGA \\
cofilin F & GAT GCT GCC AGA TAA GGA CTG \\
cofilin R & GCA ATT CAT GCT TGA TCC CTG \\
Filamin F & AGCC TCCACGAGACATCATC \\
Filamin R & CCAG TGTGTACTCCCCCTG \\
GAPDH F & GGAGATTACTGCCCTGGCTCCTAGC \\
GAPDH R & GGCCGGACTCATCGTACTCCTGCTT \\
\hline
\end{tabular}

The inflammation-affected or fibrosis-affected area in each region was counted in a high-powered field $(\times 400)$ by an investigator who was blinded to the studies and expressed as the percentage of inflammation-affected or fibrosisaffected area/total area.

Immunohistochemical staining Deparaffinized rat heart tissue sections were incubated with $3 \%$ hydrogen peroxide, and then incubated with primary antibodies to C/EBP $\beta$ (1: 1000; Santa Cruz Biotechnology, Dallas, TX), MMP-2 (1: 200, Santa Cruz Biotechnology), MMP-9 (1:200, Santa Cruz Biotechnology), Col I (1:200, Santa Cruz Biotechnology), Col III (1:200, Santa Cruz Biotechnology), and o-SMA (1:200, Santa Cruz Biotechnology). Subsequently, sections were incubated with a goat anti-mouseconjugated peroxidase-labeled secondary antibody (1: 1000; Santa Cruz Biotechnology) for 30 minutes at room temperature. Positive signals were visualized using a diaminobenzidine substrate chromagen solution and hematoxylin counterstain.

In vitro study:

Immunofluorescence microscopy analyses CMF were rinsed 3 times with PBS and fixed with $4 \%$ paraformaldehyde and subjected to $0.1 \%$ Triton X-100 (Beyotime Biotechnology, Shanghai, China) in PBS for 20 minutes at $20^{\circ} \mathrm{C}$. Cells were then washed 3 times with PBS and incubated with antibodies against cofilin (1:50; Boster, Wuhan, China) and FLNA (1:50; Sangon, Shanghai, China). Fluorophore-tagged secondary antibodies and a fluorescent microscope (Olympus BX61) were utilized for protein visualization and imaging. The fluorescence intensity was analyzed using Image-pro plus 6.0 (Media Cybernetics).

Quantitative RT-PCR analysis of C/EBP $\beta$, cofilin and FLNA mRNA Total RNA was extracted from CMF using TRIzol (Life Technologies, Rockville, MD) according to the manufacturer's protocol. cDNA was produced using M-MLV Reverse Transcriptase (Promega) according to the manufacturer's recommendations for oligo (dT) 20 or random hexamer primed cDNA-synthesis. cDNA synthesis was performed using $1 \mu \mathrm{g}$ of RNA at $40^{\circ} \mathrm{C}$. Finally, cDNA was diluted 1:5 before use in qPCR. Samples were then subjected to a quantitative polymerase chain reaction using a 7300 Real-Time PCR system (Applied Biosystems) and specific SYBR green primers (Table I; Invitrogen Life Technologies, Carlsbad, CA, USA), according to the manufacturer's protocols. Samples underwent the following PCR reaction conditions, which included an initial denaturation stage $\left(45^{\circ} \mathrm{C}, 2\right.$ minutes; $95^{\circ} \mathrm{C}, 10$ minutes $)$ followed by 40 cycles, each cycle containing 3 steps (denaturation $95^{\circ} \mathrm{C}, 15 \mathrm{~s}$; annealing $60^{\circ} \mathrm{C}, 1$ minutes; and ex- tension $72^{\circ} \mathrm{C}, 1$ minutes). Glyceraldehyde phosphate dehydrogenase (GAPDH) was evaluated as an internal control and the quantification of C/EBP- $\beta$, FLNA and cofilin mRNA relative to GAPDH was assessed. Each reaction was analyzed in triplicate.

Western blot analysis of C/EBP $\beta$, LAP, LIP and collagen Protein concentrations of the samples were determined using the Lowry method. ${ }^{24)}$ Proteins $(50 \mu \mathrm{g})$ were separated by $12 \% \mathrm{SDS}$-polyacrylamide gel and transferred onto nitrocellulose membranes, which were blocked with TBST buffer containing 5\% skim milk. Membranes were then incubated with primary antibodies directed against $\mathrm{C}$ / EBP $\beta$ (1:500; Santa Cruz Biotechnology), LAP isoform (1:500; Santa Cruz Biotechnology), and LIP isoform (1: 500; Santa Cruz Biotechnology) overnight at $4^{\circ} \mathrm{C}$. The appropriate HRP-conjugated secondary antibodies (Wanleibio, China) were used to amplify the signals of primary antibodies. Then the ECL western blotting detection reagent (Wanleibio, China) was used to display the content of protein and quantified by Image J Software.

Enzyme-linked immunosorbent assay (ELISA) The levels of inflammatory mediators were quantified using specific ELISA kits for each group (Control, Mock, Vector and Lenti-shC/EBP $\beta$ group) according to the manufacturers' protocols (tumor necrosis factor alpha (TNF-a), interleukin-1 $\beta$ (IL-1 $\beta$ ), interleukin-6 (IL-6), from Merck Millipore, MA, USA).

Statistical Analysis All data are expressed as the mean \pm standard deviation. Statistical comparisons were performed using one-way analysis of variance (ANOVA) followed by Tukey's post hoc test for multiple comparison (Graphpad Prism, version 5.0). $P$ values of less than 0.05 were considered statistically significant.

\section{Results}

\section{In vitro:}

Lenti-viral shRNA approaches are sufficient to significantly reduce C/EBP $\beta$ mRNA and protein expression in $\overline{C M F}$ To investigate the role of C/EBP $\beta$ in CF differentiation, we used a lentivirus harboring $\mathrm{C} / \mathrm{EBP} \beta$ shRNA (Lenti-shC/EBP $\beta$ ). Quantitative RNA analysis of TGF- $\beta 1$ treated $\mathrm{CF}$ that were infected with Lenti-shC/EBP $\beta$ resulted in significant reductions in $\mathrm{C} / \mathrm{EBP} \beta$ mRNA, cofilin mRNA, and FLNA mRNA levels compared to the control (control, mock and vector) groups (Figure 2). Protein blot analyses of CMF infected with Lenti-shC/EBP $\beta$ also demonstrated significant decreases in C/EBP $\beta$ - (LAP+LIP), C/ $\mathrm{EBP} \beta$-LAP, and $\mathrm{C} / \mathrm{EBP} \beta$-LIP protein expression (Figure $3)$.

C/EBP $\beta$ knockdown could attenuate cytoskeletal remodeling in $C M F$ To determine the role of C/EBP $\beta$ on cytoskeletal switching and remodeling in $\mathrm{CMF}, \mathrm{CMF}$ were infected with Lenti-shC/EBP $\beta$. We observed that $\mathrm{C} / \mathrm{EBP} \beta$ knockdown significantly attenuated cofilin and FLNA mRNA expression in CMF when compared with controls (not infected with Lenti-shC/EBP $\beta$ ) (Figure 4). Thus, knockdown of $\mathrm{C} / \mathrm{EBP} \beta$ can attenuate actin cytoskeletal remodeling in $\mathrm{CMF}$ in vitro.

C/EBP $\beta$ knockdown could reduce inflammatory markers As shown in Table II, there was a marked increase in the 
levels of the inflammatory markers IL- $1 \beta$, IL-6, and TNF$a$ in the Mock group and the Vector group compared with the Control group. Administration of Lenti-C/EBP $\beta$ reduced the above-mentioned inflammatory mediators (Table II).

In vivo:

$C / E B P \beta$ knockdown attenuated cardiac inflammation and fibrosis in EAM rats To determine the role of C/EBP $\beta$ on cardiac remodeling in a rat model of EAM, rats were injected with Lenti-shC/EBP $\beta$ in vivo and histopathological analyses were performed (Figure 5).

$C / E B P \beta$ knockdown attenuated fibrotic markers in EAM rat hearts We performed immunohistochemical assessment

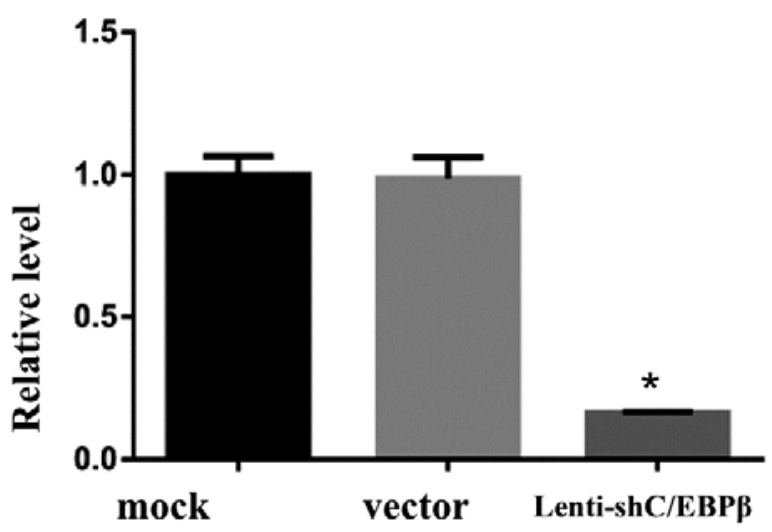

Figure 2. Assessing expression of C/EBP $\beta$ by quantitative RT-PCR in vitro. The effects of treatment with a lentivirus containing an shRNA sequence targeting C/EBP $\beta$ expression in cells was tested by quantitative RT-PCR. Relative RNA expression of C/EBP $\beta$ (LIP + LAP) in CMFs was performed after Lentiviral transfection. Values were normalized to the mean of the Control group and are expressed as arbitrary units. The lentivirus reduced expression of C/EBP $\beta$ in the LentishC/EBP $\beta$ group. Mock group indicates the TGF $\beta 1$ group. Vector group indicates the TGF $\beta 1+$ Lentiviral Vector group; Lenti-shC/EBP $\beta$ indicates the TGF- $\beta 1+$ Lenti-shC/EBP $\beta$ group. ${ }^{*} P<0.05$ versus the Mock group and the Vector group. of collagen I, collagen III, MMP-2, MMP-9, $\alpha$-SMA, and $\mathrm{C} / \mathrm{EBP} \beta$ in rat heart tissues. We observed that collagen I, collagen III, MMP-2, MMP-9, $\alpha$-SMA, and C/EBP $\beta$ protein expressions are increased in heart tissue sections from EAM rats (EAM group and EAM + Lentiviral Vector group), when compared to the Control group (Figure 6). Furthermore, $\mathrm{C} / \mathrm{EBP} \beta$ knockdown attenuated the $\mathrm{C} / \mathrm{EBP} \beta$ protein expression as well as expression of collagen I, collagen III, MMP-2, MMP-9, and $\alpha$-SMA in heart tissue sections from rats in the EAM + Lenti-shC/EBP $\beta$ group (Figure 6). These results indicate that $\mathrm{C} / \mathrm{EBP} \beta$ knockdown is capable of dampening inflammation mediated induction of $\alpha$-SMA and collagen production, suggesting that $\mathrm{C} /$ EBP $\beta$ is critical in myofibroblast differentiation.

C/EBP $\beta$ knockdown improved cardiac dimensions and function in EAM rats To determine the role of C/EBP $\beta$ on heart structure and function in the rat EAM model, we performed echocardiography on rats in the Control, EAM, $\mathrm{EAM}+$ Lentiviral Vector, and EAM+Lenti-shC/EBP $\beta$ groups. We observed cardiac hypertrophy, wall thinning, and cardiac dysfunction in rats in the EAM and EAM+ Lentiviral Vector groups. However, C/EBP $\beta$ knockdown could significantly improve cardiac dimensions and function in rats in the EAM+ Lenti-shC/EBP $\beta$ group (Table III).

\section{Discussion}

Myocarditis is a pivotal cause of sudden death among young adults. ${ }^{26)}$ It is also often the vaunt-courier of cardiac remodeling and heart failure. The rat EAM model is one that recapitulates immuno-inflammatory cardiac remodeling (fibrosis). ${ }^{27)}$ Myocardial fibrosis plays a principal role in the pathophysiology of heart failure; however, the mechanisms driving inflammation-mediated cardiac fibrosis remain unclear. $\mathrm{C} / \mathrm{EBP} \beta$ is an important regulator of genes involved in immune and inflammatory responses, but recent evidence also suggests that its loss can attenuate fibrosis in the lung and liver, ${ }^{17,18)}$ highlighting its po-
A

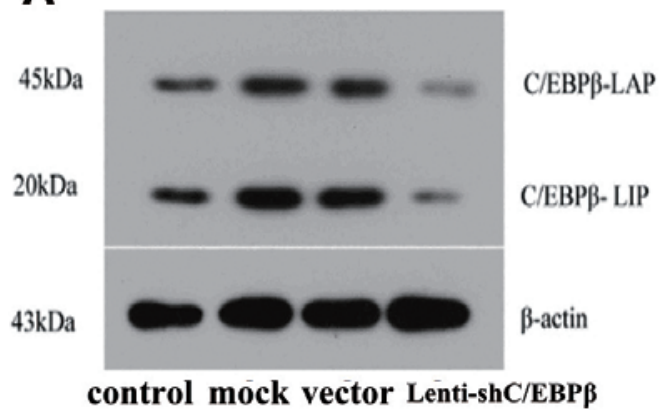

B

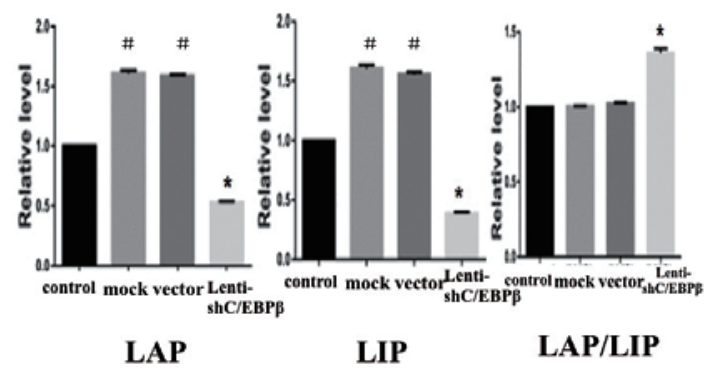

Figure 3. Assessing the expression of C/EBP $\beta$ by Western blot analysis. The protein expression of C/EBP $\beta$ (LAP and LIP), LAP, and LIP was measured by Western blot in cultured CMFs and the ratio of LAP/LIP was calculated. In the Lenti-shC/ EBP $\beta$ group, the expressions of C/EBP $\beta$, LAP, and LIP were all lower than that of the Control group, Mock group, and Vector group. The LAP/LIP ratio was increased significantly. Densitometric analysis was performed to quantify the protein levels. Values were normalized to the mean of the Control group and expressed as arbitrary units. A and B: Representative Western blot analyses and bar graphs of LAP, LIP, and LAP/LIP. Control group indicates CFs with no treatment. Mock group indicates TGF $\beta 1$ group. Vector indicates TGF $\beta 1+$ Lentiviral Vector group; Lenti-shC/EBP $\beta$ group indicates TGF- $\beta 1+$ Lenti-shC/EBP $\beta$ group. $* P<0.05$ versus Control group, Mock group and Vector group. ${ }^{\#} P<0.05$ versus Control group and Lenti-shC/EBP $\beta$ group. 


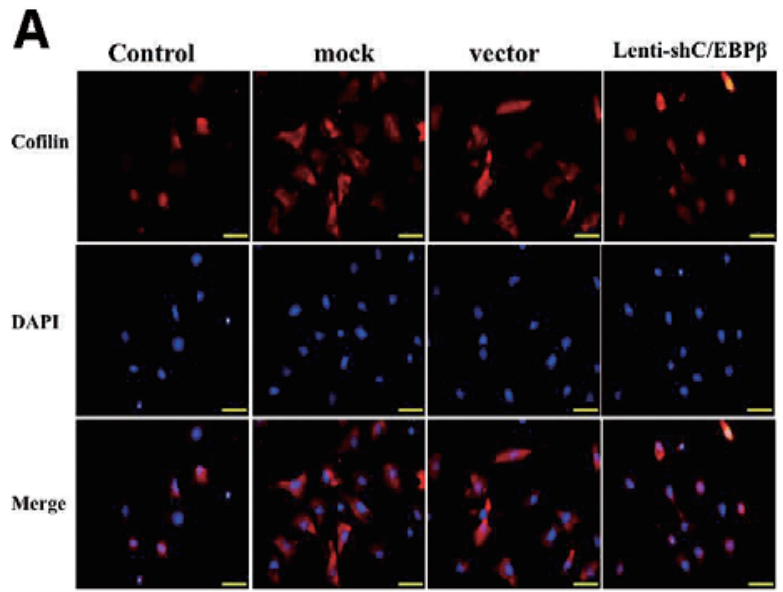

B
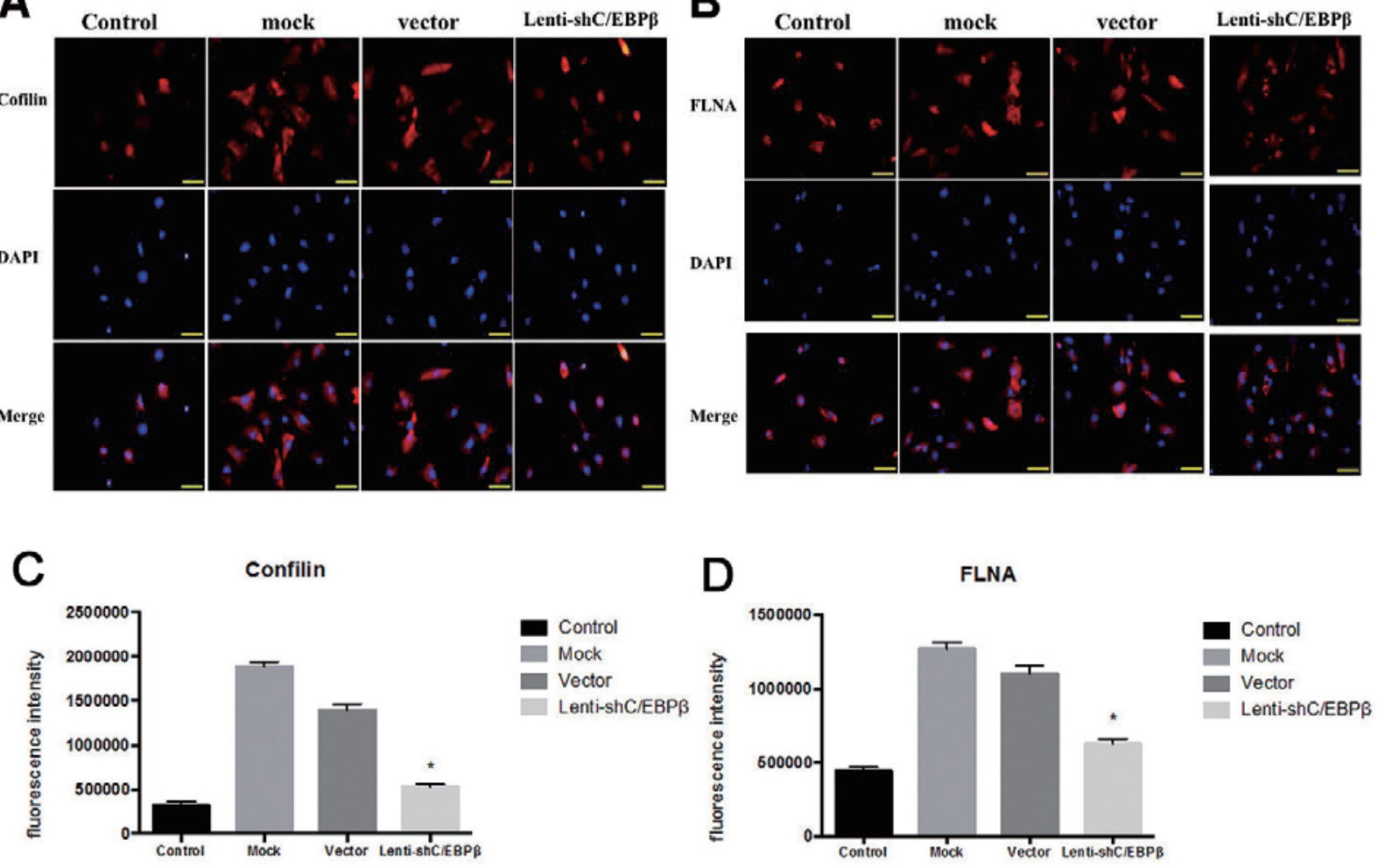

\section{E Quantitative RT-PCR results of cofilin and FLNA}

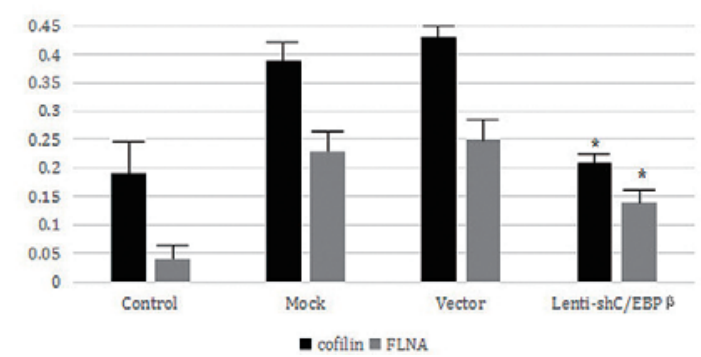

Figure 4. Assessing the level of cofilin and FLNA expression in cells. Immunofluorescence staining was used to measure the expression of cofilin and FLNA in vitro for 24 hours. Both cofilin and FLNA in the TGF $\beta 1+$ Lenti-shC/EBP $\beta$ group are lower than the Control group and TGF- $\beta 1$ group $(P<0.05)$. A: Cofilin and nuclei were stained with Cy5 (red) and DAPI (blue), respectively. B: FLNA and nuclei were stained with Cy5 (red) and DAPI (blue), respectively. TGF $\beta 1+$ CL indicates TGF $\beta 1+$ Lentiviral Vector group; TGF $\beta 1$ + EL indicates TGF- $\beta 1$ + LentishC/EBP $\beta$ group. $\mathbf{C}$ and D: The fluorescence intensity of cofilin and FLNA. Values were normalized to the mean of Control group and expressed as arbitrary units. ${ }^{*} P<0.05$ versus the Mock and Vector groups. E: Quantitative RT-PCR results of Cofilin and FLNA. Values were normalized to the mean of Control group and expressed as arbitrary units. $* P<0.05$ versus the Mock and Vector groups.

Table II. The Levels of Inflammatory Mediators Measured by ELISA in Each Group in the in vitro Study

\begin{tabular}{lcll}
\hline \multicolumn{1}{c}{ Group } & $\mathrm{IL}-1 \beta(\mathrm{pg} / \mathrm{mL})$ & \multicolumn{1}{c}{$\mathrm{IL}-6(\mathrm{pg} / \mathrm{mL})$} & TNF-a $(\mathrm{pg} / \mathrm{mL})$ \\
\hline Control & $48.761 \pm 4.540$ & $154.662 \pm 14.321$ & $22.561 \pm 6.561$ \\
Mock & $376.650 \pm 10.211^{*}$ & $606.383 \pm 13.331^{*}$ & $98.322 \pm 7.353^{*}$ \\
Vector & $362.190 \pm 12.833^{*}$ & $712.312 \pm 21.863^{*}$ & $92.091 \pm 9.933^{*}$ \\
Lenti-C/EBP $\beta$ & $68.420 \pm 7.092$ & $202.154 \pm 18.921$ & $36.771 \pm 5.291$ \\
\hline
\end{tabular}

TNF-a indicates tumor necrosis factor alpha $(\mathrm{pg} / \mathrm{mL})$; IL-1 $\beta$, interleukin-1 $\beta(\mathrm{pg} / \mathrm{mL})$; and IL-6, interleukin- $6(\mathrm{pg} / \mathrm{mL}) . * P<0.05$ versus the Control group and the Lenti-C/ $\mathrm{EBP} \beta$ group. 

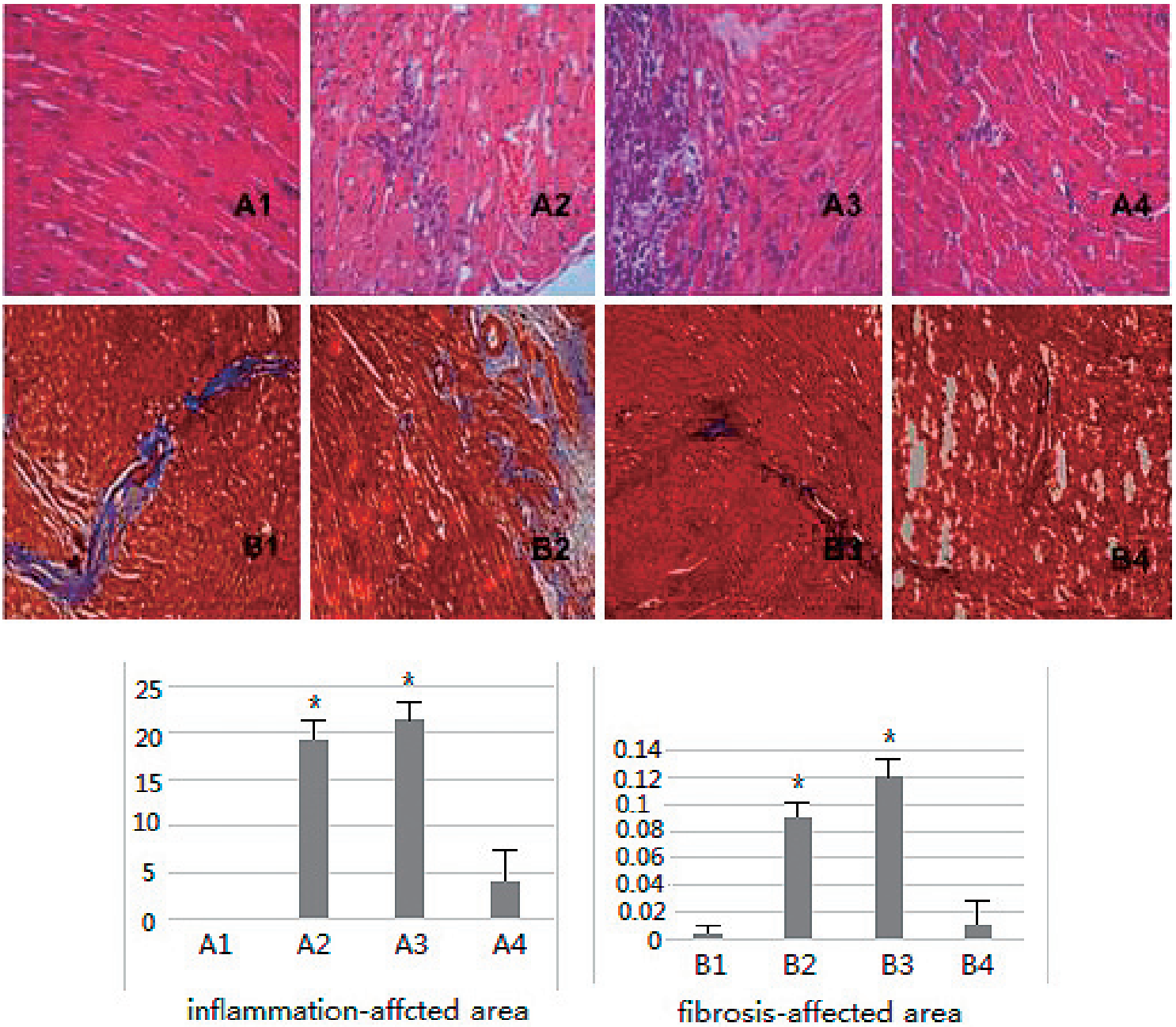

Figure 5. Representative images of HE and Masson's staining of the heart tissue. A1: Control group (HE staining; original magnification $200 \times$ ). A2: EAM group (HE staining; original magnification $200 \times$ ). A3: EAM + Lentiviral Vector group (HE staining; original magnification $200 \times$ ). A4: EAM + Lenti-shC/EBP $\beta$ group (HE staining; original magnification $200 \times$ ). B1: Control group, B2: EAM group, B3: EAM + Lentiviral Vector group, and B4: EAM + Lenti-shC/EBP $\beta$ group. Total magnification: $200 \times$. Values were normalized to the mean of Control group and expressed as arbitrary units. $* P<0.05$ versus the control group and the Lenti-shC/EBP $\beta$ group.

tential as a novel therapeutic target for regulating the immuno-inflammatory tissue fibrotic response.

TGF- $\beta 1$ is a potent driver of myofibroblast differentiation from various progenitor cells, primarily resident fibroblasts. The findings of this study suggest that TGF- $\beta 1$ mediated myofibroblast differentiation is sensitive to changes of $\mathrm{C} / \mathrm{EBP} \beta$ knockdown as shown by the reduction of actin regulatory protein expression. Cofilin and FLNA are newly discovered members of the actin regulatory protein family. ${ }^{27,28)}$ FLNA binds to F-actin and induces high-angle crosslinking to provide a strong 3dimensional cytoskeletal structure. ${ }^{29-31)}$ Studies have described the importance of actin polymerization/depolymerization as key mediators of CMF phenotype transformation. $^{32,33)}$ Specifically, polymerization/depolymerization of $\alpha$-SMA is controlled by the actin depolymerizing factor (ADF)/cofilin family, which binds polymerized actin (Factin) and breaks it down into G-actin by depolymerization, thereby regulating the stability and function of $\alpha$ -
SMA. ${ }^{34)}$ We found that $\mathrm{C} / \mathrm{EBP} \beta$ knockdown could reduce the protein expression of cofilin and FLNA, suggesting that the cofilin/FLNA signaling pathway may be a downstream effector of $\mathrm{C} / \mathrm{EBP} \beta$ during $\mathrm{CF}$ differentiation.

$\mathrm{C} / \mathrm{EBP} \beta$ is an intronless gene that codes for the production of a single mRNA. The C/EBP $\beta$ mRNA transcript can translate into 4 different protein isoforms: full length $\mathrm{C} / \mathrm{EBP} \beta$ or $\mathrm{LAP}^{*}$ (liver-enriched transcriptional activating protein star) with an atomic mass of $38 \mathrm{kDa}$, LAP (liverenriched transcriptional activating protein), which has an atomic mass of $35 \mathrm{kDa}$, the $20 \mathrm{kDa}$ isoform LIP (liverenriched transcriptional inhibitory protein), and a smaller $16 \mathrm{kDa}$ isoform. The isoform LAP is a transcriptional activator, while the isoform LIP, which lacks the transactivation domain, is a transcriptional inhibitor. ${ }^{35}$ C/EBP $\beta$ mRNA can be translated into two isoforms, which include LAP (LAP*) and LIP. LIP lacks the activation domain and is thus thought to be the dominant negative isoform of LAP ${ }^{36)}$ The ratio of LAP/LIP was considered as an in- 
A
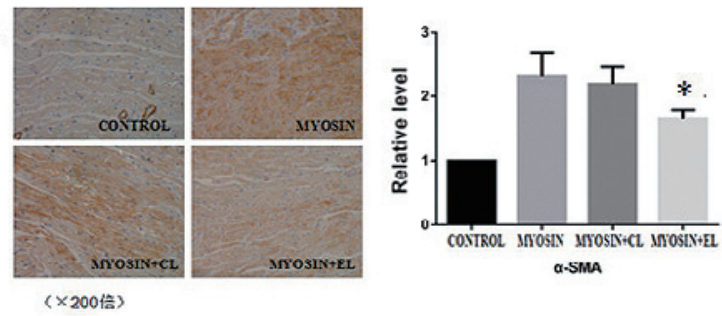

C

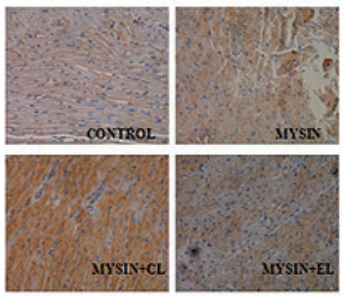

$(\times 200$ 信 $)$

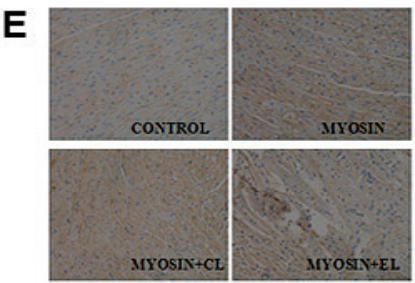

( $\times 200$ 信)
B

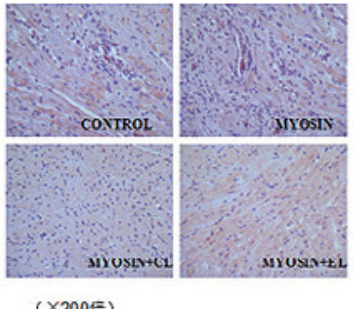

( $\times 200$ 借)

D
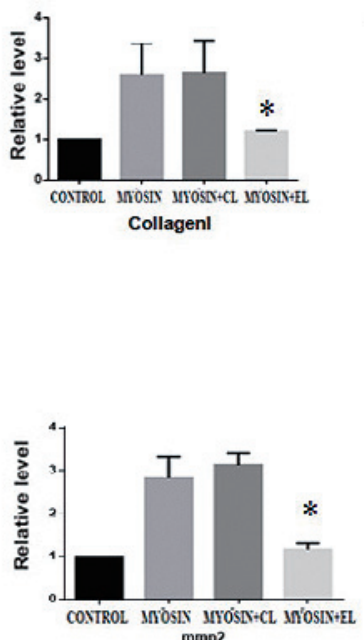

mmp2

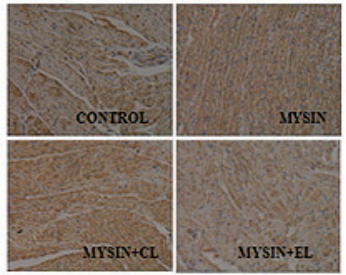

( $\times 200$ 倍)

F

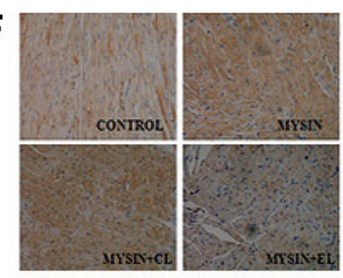

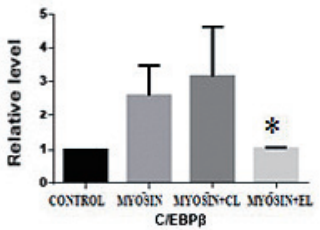

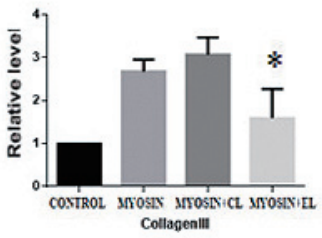

Collagenill

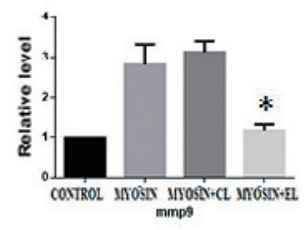

( $\times 200$ 佮)

Figure 6. Cardiac fibrosis was attenuated in the knockdown of C/EBP $\beta$. Quantification of $\alpha$-smooth muscle action ( $\alpha$-SMA), C/EBP $\beta$, collagen I, collagen III, matrix metalloproteinase (MMP)-2, and MMP-9 in the rats' heart tissue. Specific epitopes of $\alpha$-SMA, C/EBP $\beta$, collagen I, collagen III, and MMP-2, MMP-9 are colored brown. Quantification was performed by digital image analyses. Values were normalized to the mean of Control group and expressed as arbitrary units. ${ }^{*} P<0.05$ versus the EAM group and EAM + Lentiviral Vector group.

Table III. Echocardiography Results

\begin{tabular}{llllllc}
\hline \multicolumn{1}{c}{ Group } & \multicolumn{1}{c}{ IVS $(\mathrm{mm})$} & LVPW $(\mathrm{mm})$ & \multicolumn{1}{c}{ LVEDD $(\mathrm{mm})$} & LVESD $(\mathrm{mm})$ & \multicolumn{1}{c}{ LVEF $(\%)$} & FS $(\%)$ \\
\hline Control & $1.320 \pm 0.031$ & $1.273 \pm 0.101$ & $4.974 \pm 0.172$ & $2.781 \pm 0.570$ & $82.152 \pm 5.132$ & $45.633 \pm 5.342$ \\
EAM & $1.121 \pm 0.093$ & $1.114 \pm 0.071$ & $5.910 \pm 0.182$ & $3.922 \pm 1.071$ & $63.924 \pm 5.232$ & $32.281 \pm 9.241$ \\
EAM + Lentiviral Vector & $1.140 \pm 0.034$ & $1.101 \pm 0.031$ & $6.063 \pm 0.063$ & $4.141 \pm 0.112$ & $65.033 \pm 3.210$ & $32.572 \pm 2.471$ \\
EAM + Lenti-shC/EBP 3 & $1.283 \pm 0.051^{*}$ & $1.243 \pm 0.070^{*}$ & $5.490 \pm 0.182^{*}$ & $3.501 \pm 0.780^{*}$ & $79.332 \pm 2.170$ & $43.322 \pm 2.390^{*}$ \\
\hline
\end{tabular}

Assessing cardiac function by echocardiography in a rat model. Data are presented as the mean \pm SD. IVS indicates interventricular septal thickness; LVPW, left ventricular posterior wall; LVEDD, left ventricular ejection diameter; LVEF, left ventricular ejection fraction; and FS, fractional shortening. $* P<0.05$ versus the EAM + Lentiviral Vector group and EAM group.

dicator for C/EBP $\beta$ activity. Our studies demonstrated an increase in the LAP/LIP ratio in C/EBP $\beta$ knockdown studies. It is possible that although both LAP and LIP isoforms were targeted by the C/EBP $\beta$ shRNA lentivirus; that LIP may be more impacted than LAP. The C/EBP $\beta$ lentivirus inhibits LAP as well as LIP. These two come from the same mRNA. However, because the molecular activity of LIP is much higher than LAP, the degree of inhibition of LIP would be more significant than that of LAP. Therefore, sh-C/EBP $\beta$ impacts LIP more than LAP. $^{37)}$ Some studies have demonstrated that increasing LIP isoform expression may have the capacity to control LAP activity, resulting in a decrease in the LAP/LIP ratio, and decreased $\alpha$-SMA expression. ${ }^{38)}$

Recently, it has been found that the regulation of a-
SMA expression and polymerization is the key to the phenotypic transformation from fibroblasts into myofibroblasts. ${ }^{10)}$ These processes are regulated by serum response factor (SRF). The actin gene regulatory region of a-SMA contains the CArG box element. ${ }^{39,40)}$ SRF regulates a-SMA through this regulatory element. ${ }^{41)}$ In unpublished results, we found the pretreatment of $\mathrm{CF}$ with the SRF blocker CCG-1423 could significantly inhibit C/EBP $\beta$ induced CF differentiation. This suggested that SRF may be involved in $\mathrm{C} / \mathrm{EBP} \beta$ induced $\mathrm{CF}$ transformation. The SRF gene regulatory region contains CCAAT binding element, which specifically binds to $\mathrm{C} / \mathrm{EBP} \beta$ and regulates the expression of target gene. ${ }^{42)} \mathrm{SRF}$ is a weak transcriptional activator, which plays a role in the regulation of gene expression. It can promote the transcription factor to form 
the complex, and thus initiate transcription of the target gene. ${ }^{43)}$ The dependence on transcription factors is the basic action model of SRF. We speculate that transcription factor complex $\mathrm{C} / \mathrm{EBP} \beta-\mathrm{SRF}$ plays an important role in differentiation from $\mathrm{CF}$ into $\mathrm{CMF}$. However, further study is needed to verify the possible interactions between $\mathrm{C} /$ $\mathrm{EBP} \beta$ and SRF protein, and the effects of this interaction on the expression of a-SMA and its polymerization and depolymerization.

Cardiac fibroblasts play a crucial role in extracellular matrix regulation as they produce structural proteins that comprise the extracellular matrix, such as collagen, as well as promote their degradation by secreting MMP proteins. We provide evidence that $\mathrm{C} / \mathrm{EBP} \beta$ knockdown in a rat EAM model significantly attenuated cardiac collagen I, collagen III, MMP-2, and MMP-9 protein levels. C/EBP $\beta$ knockdown also impacted the fibroblasts inflammatory response as indicated by IL-1 $\beta$, IL- 6 , and TNF-a production. It is well established that members of the C/EBP family regulate target genes associated with inflammation, including many cytokines and chemokines. ${ }^{17,39,40)}$ Our studies also demonstrate that $\mathrm{C} / \mathrm{EBP} \beta$ knockdown resulted in a cardiac structural and functional improvement in the rat EAM model. Previous studies have indicated that $\mathrm{C} / \mathrm{EBP} \beta$ heterozygous deficient mice displayed improved cardiac function in the face of pressure overload, ${ }^{41)}$ which is consistent with our study findings.

Taken together, our findings show that $\mathrm{C} / \mathrm{EBP} \beta$ is a positive regulator of cardiac inflammation and fibrosis in EAM rats. We have demonstrated that $\mathrm{C} / \mathrm{EBP} \beta$ regulates the actin remodeling proteins cofilin and FLNA in vitro, which are impacted during CMF differentiation. These findings may impact our in vivo findings utilizing $\mathrm{C} / \mathrm{EBP} \beta$ shRNA lentiviral approaches in the rat EAM model, which demonstrate a role for $\mathrm{C} / \mathrm{EBP} \beta$ in extracellular matrix production and degradation. Thus, the cofilin and FLNA-related signaling pathways may provide a potential new therapeutic target to attenuate cardiac fibrosis. However, additional future studies are warranted to investigate the detailed mechanisms by which $\mathrm{C} / \mathrm{EBP} \beta$ attenuates cardiac fibrosis.

\section{Disclosures}

\section{Conflicts of interest: None.}

\section{References}

1. Luo YX, Tang X, An XZ, et al. Sirt4 accelerates Ang II-induced pathological cardiac hypertrophy by inhibiting manganese superoxide dismutase activity. Eur Heart J 2017; 38: 1389-98.

2. Rommel KP, von Roeder M, Latuscynski K, et al. Extracellular volume fraction for characterization of patients with heart failure and preserved ejection fraction. J Am Coll Cardiol 2016; 67: $1815-25$

3. Zhou J, Gao J, Zhang X, Liu Y, et al. microRNA-340-5p functions downstream of cardiotrophin-1 to regulate cardiac eccentric hypertrophy and heart failure via target gene dystrophin. Int Heart J 2015; 56: 454-8.

4. Hayami N, Sekiguchi A, Iwasaki YK, Murakawa Y, Yamashita T. No additional effect of DPP-4 inhibitor on preventing atrial fi- brosis in streptozotocin-induced diabetic rat as compared with sulfonylurea. Int Heart J 2016; 57: 336-40.

5. Blyszczuk P, Müller-Edenborn B, Valenta T, et al. Transforming growth factor- $\beta$-dependent Wnt secretion controls myofibroblast formation and myocardial fibrosis progression in experimental autoimmune myocarditis. Eur Heart J 2017; 38: 1413-25.

6. Ghosh AK, Nagpal V, Covington JW, Michaels MA, Vaughan DE. Molecular basis of cardiac endothelial-to-mesenchymal transition (EndMT): Differential expression of microRNAs during EndMT. Cell Signal 2012; 24: 1031-6.

7. Ding Z, Yuan J, Liang Y, et al. Ryanodine receptor type 2 plays a role in the development of cardiac fibrosis under mechanical stretch through TGF $\beta-1$. Int Heart J 2017; 58: 957-61.

8. Mani SK, Kern CB, Kimbrough D, et al. Inhibition of class I histone deacetylase activity represses matrix metalloproteinase-2 and -9 expression and preserves LV function postmyocardial infarction. Am J Physiol Heart Circ Physiol 2015; 308: H1391401.

9. Bargagna-Mohan P, Deokule SP, Thompson K, et al. Withaferin A effectively targets soluble vimentin in the glaucoma filtration surgical model of fibrosis. PLoS One 2013; 8: e63881.

10. Ghosh AK, Nagpal V, Covington JW, Michaels MA, Vaughan DE. Molecular basis of cardiac endothelial-to-mesenchymal transition (EndMT): Differential expression of microRNAs during EndMT. Cell Signal 2012; 24: 1031-6.

11. Yamakawa H, Ieda M. Strategies for heart regeneration: approaches ranging from induced pluripotent stem cells to direct cardiac reprogramming. Int Heart J 2015; 56: 1-5.

12. Kanno Y, Watanabe R, Zempo H, Ogawa M, Suzuki J, Isobe M. Chlorogenic acid attenuates ventricular remodeling after myocardial infarction in mice. Int Heart J 2013; 54: 176-80.

13. Wei L. Immunological aspect of cardiac remodeling: T lymphocyte subsets in inflammation-mediated cardiac fibrosis. Exp Mol Pathol 2011; 90: 74-8.

14. Tate M, Robinson E, Green BD, McDermott BJ, Grieve DJ. Exendin-4 attenuates adverse cardiac remodeling in streptozocin-induced diabetes via specific actions on infiltrating macrophages. Basic Res Cardiol 2016; 111: 1.

15. Ramji DP, Foka P. CCAAT/enhancer-binding proteins: structure, function and regulation. Biochem J 2002; 365: 561-75.

16. Manea SA, Todirita A, Raicu M, Manea A. C/EBP transcription factors regulate NADPH oxidase in human aortic smooth muscle cells. J Cell Mol Med 2014; 18: 1467-77.

17. Liu W, Li S, Tian W, Li W, Zhang Z. Immunoregulatory effects of $\alpha$-GalCer in a murine model of autoimmune myocarditis. Exp Mol Pathol 2011; 91: 636-42.

18. Hu B, Ullenbruch MR, Jin H, Gharaee-Kermani M, Phan SH. An essential role for CCAAT/enhancer binding protein beta in bleomycin-induced pulmonary fibrosis. J Pathol 2007; 211: 45562.

19. Zeng QC, Guo Y, Liu L, et al. Cardiac fibroblast-derived extracellular matrix produced in vitro stimulates growth and metabolism of cultured ventricular cells. Int Heart J 2013; 54: 40-4.

20. Chen ZY, Lin Y, Yang F, Jiang L, Ge Sp. Gene therapy for cardiovascular disease mediated by ultrasound and microbubbles. Cardiovasc Ultrasound 2013; 11: 11.

21. Wei L, Wei-Min L, Cheng G, Bao-Guo Z. Upregulation of CD 41CD251 T lymphocyte by adenovirus-mediated gene transfer of CTLA4Ig fusion protein in experimental autoimmune myocarditis. Autoimmunity 2006; 39: 289-98.

22. Xie D, Liao Y, Wu B, et al. Cardiac Nestin ${ }^{+}$cells derived from early stage of dilated cardiomyopathy enhanced the survival of Doxorubicin-injured cardiac muscle HL-1 cells. Int Heart J 2018; 59: 180-9.

23. Nishina T, Nishimura K, Yuasa S, et al. Initial effects of the left ventricular repair by plication may not last long in a rat ischemic cardiomyopathy model. Circulation 2001; 104: I241-5.

24. Lowry DH, Rosenberg NJ, Farr AL, Randall RJ. Protein measurement with folin phenol reagent. J Biol Chem 1951; 193: $265-75$. 
25. Zeng QC, Guo Y, Liu L, et al. Cardiac fibroblast-derived extracellular matrix produced in vitro stimulates growth and metabolism of cultured ventricular cells. Int Heart J 2013; 54: 40-4.

26. Li WM, Liu W, Gao C, Zhou BG. Immunoregulatory effects of atorvastatin on experimental autoimmune myocarditis in Lewis rats. Immunol Cell Biol 2006; 84: 274-80.

27. Liu W, Feng W, Wang F, et al. Osteoprotegerin/RANK/RANKL axis in cardiac remodeling due to immuno-inflammatory myocardial disease. Exp Mol Pathol 2008; 84: 213-7.

28. Kim JI, Kwon J, Baek I, Na S. Steered molecular dynamics analysis of the role of cofilin in increasing the flexibility of actin filaments. Biophys Chem 2016; 218: 27-35.

29. Winterhoff M, Faix J. Actin-filament disassembly: it takes two to shrink them fast. Curr Biol 2015; 25: R450-2.

30. Gressin L, Guillotin A, Guérin C, Blanchoin L, Michelot A. Architecture dependence of actin filament network disassembly. Curr Biol 2015; 25: 1437-47.

31. Hayakawa K, Sakakibara S, Sokabe M, Tatsumi H. Singlemolecule imaging and kinetic analysis of cooperative cofilinactin filament interactions. Proc Natl Acad Sci USA 2014; 111: 9810-5.

32. Bamburg JR, Bernstein BW. Actin dynamics and cofilin-actin rods in Alzheimer disease. Cytoskeleton (Hoboken) 2016; 73: 477-97.

33. Nomura K, Hayakawa K, Tatsumi H, Ono S. Actin-interacting protein 1 promotes disassembly of actin-depolymerizing factor/ cofilin-bound actin filaments in a pH-dependent manner. J Biol Chem 2016; 291: 5146-56.

34. Butkevich E, Bodensiek K, Fakhri N, et al. Drebrin-like protein DBN-1 is a sarcomere component that stabilizes actin filaments during muscle contraction. Nat Commun 2015; 6: 7523.

35. Van der Krieken SE, Popeijus HE, Mensink RP, Plat J. CCAAT/ enhancer binding protein $\beta$ in relation to ER stress, inflammation, and metabolic disturbances. Biomed Res Int 2015; 2015: 324815.

36. Huber R, Panterodt T, Weiz B, et al. C/EBP $\beta-L A P * / L A P ~ E x-$ pression Is Mediated by RSK/eIF4B-Dependent Signalling and Boosted by Increased Protein Stability in Models of Monocytic Differentiation. PLoS One 2015; 10: e0144338.
37. Buck M, Turler H, Chojier M. LAP (NF-IL-6), a tissue-specific transcriptional activator, is an inhibitor of hepatoma cell proliferation. EMBO J 1994; 13: 851-60.

38. Hu B, Wu Z, Jin H, Hashimoto N, Liu T, Phan SH. CCAAT/ enhancer-binding protein beta isoforms and the regulation of alpha-smooth muscle actin gene expression by IL-1 beta. J Immunol 2004; 173: 4661-8.

39. Aoyagi-Ikeda K, Maeno T, Matsui $\mathrm{H}$, et al. Notch induces myofibroblast differentiation of alveolar epithelial cells via transforming growth factor- $\beta$-Smad3 pathway. Am J Respir Cell Mol Biol 2011; 45: 136-44.

40. Verdoni AM, Schuster KJ, Cole BS, Ikeda A, Kao WW, Ikeda S. A pathogenic relationship between a regulator of the actin cytoskeleton and serum response factor. Genetics 2010; 186: 14757.

41. Sekinishi A, Suzuki J, Aoyama N, et al. Periodontal pathogen aggregatibacter actinomycetemcomitans deteriorates pressure overload-induced myocardial hypertrophy in mice. Int Heart $\mathbf{J}$ 2012; 53: 324-30.

42. Hanlon M, Sturgill TW, Sealy L. ERK2- and p90(Rsk2)dependent pathways regulate the CCAAT/enhancer-binding protein-beta interaction with serum response factor. J Biol Chem 2001; 276: 38449-56.

43. Luchsinger LL, Patenaude CA, Smith BD, Layne MD. Myocardin-related transcription factor-A complexes activate type I collagen expression in lung fibroblasts. J Biol Chem 2011; 286: 44116-25.

44. Poli V, Cortese R. Interleukin 6 induces a liver-specific nuclear protein that binds to the promoter of acute-phase genes. Proc Natl Acad Sci USA 1989; 86: 8202-6.

45. Ramji DP, Vitelli A, Tronche F, Cortese R, Ciliberto G. The two C/EBP isoforms, IL-6DBP/NF-IL6 and C/EBP delta/NF-IL6 beta, are induced by IL-6 to promote acute phase gene transcription via different mechanisms. Nucleic Acids Res 1993; 21: 289-94.

46. Boström P, Mann N, Wu J, et al. C/EBP $\beta$ controls exerciseinduced cardiac growth and protects against pathological cardiac remodeling. Cell 2010; 143: 1072-83. 